\title{
On Using Divide and Conquer in Modeling Natural Systems
}

\author{
Yaki Setty, Irun R. Cohen, Avi E. Mayo, \\ and David Harel
}

\begin{abstract}
In recent years, we have been studying approaches to the realistic modeling of natural systems, especially biological systems. We have tested several of these in a project devoted to modeling pancreatic organogenesis, a complex system that dynamically promotes structural and molecular development. Here, we describe one of these approaches - a kind of 'divide and conquer' technique, in which the system is disassembled into modules to specify behaviors on the scale of the organ (i.e., morphogenesis) and the cell (i.e., molecular interactions). At run-time, these modules are re-assembled to direct development in individual cells. This approach employs multi-scaling and dynamics, two important characteristics of natural systems, but avoids cross-scaling. It thus appears to be useful for systems in which the importance of cross-scaling seems to be less significant, such as the development of phyllotaxis in plants. In pancreatic organogenesis, cross-scaling was found to be a significant characteristic, and thus by using 'divide and conquer' we could cover only its preliminary stages. We discuss the approach and our use of it, as well as he various methods to analyze the achievements and limitations of the end result.
\end{abstract}

\section{Introduction}

Natural systems, such as organs and organisms, are large-scale complex systems with numerous elements and interactions. Modeling such systems can lead to better understanding thereof and may help in efforts to save on resources and development time. During the past years, we have been focused on developing approaches for modeling natural systems. We developed several approaches to simulate the natural systems through changes in 4 dimensions: time and three dimensions of space. The chain of reactions leading to cellular and structural development emphasizes the need for multi-scaling. Furthermore, the importance of 4D dynamics in natural systems underlies the time-dependent developmental processes that affect the developing organ. Thus, for the very least, a plausible approach should conduct dynamic formation of a 3D structure.

\footnotetext{
Y. Setty $(\bowtie)$

Computational Biology Group, Microsoft Research, Cambridge CB3 0FB, UK

e-mail: yaki.setty@microsoft.com
} 
We tested our approaches on pancreatic organogenesis, a complex system in which the pancreas is developed and formed. This system involves multiple scales, dynamics, and a unique 3D anatomic structure. During organogenesis, reactions in cells lead to cellular behavior and anatomic structuring of the organ. In turn, the cell's position feeds back and influences gene expression. In pancreatic organogenesis, cells act in concert and aggregate to a cauliflower-shape structure. Zooming into the formation process discloses the molecular interactions that drive each cell during its life cycle.

The end result approach, which we term autonomous cell, defines a cell as a 3D entity that senses its environment and acts accordingly. This approach was found very beneficial for modeling pancreatic organogenesis and conduct multi-scale, cross-scale, and dynamics as well as emergence of the 3D organ structure from a collection of molecular interactions. The approach enables integration of the genetic, molecular, and cellular components, along with environmental signaling into a dynamically running, three-dimensional simulation (see [40]).

However, as a step toward the autonomous cell approach, we have developed several approaches that served as important milestones in our work, and enhanced different aspects in the task of modeling natural systems. The 'divide and conquer' approach $^{1}$ that we describe here suggests decomposing a system into independent modules and reassembled them at run time. Accordingly, we defined two main modules; one specifies the structural development of the system and the other formalizes the molecular interactions in an individual cell.

In natural systems, such as pancreatic organogenesis, these two modules correspond to different scales of the system [7]. The specifications of molecular interactions correspond to behavior of the system at the cell scale, while the formation of the organ specifies the morphogenesis at the organ scale. From the biological point of view, the 'divide and conquer' approach suggests to separate scales in natural systems, namely, the behavior of the organ from the behavior of individual cells. Thus, this approach conducts multi-scaling and 3D dynamics, two important characteristics of realistic modeling [7].

To apply the 'divide and conquer' approach to pancreatic organogenesis, we formalized a eukaryotic cell as an object with a sub-object for its nucleus. We specified in a molecular interaction module the relevant interactions in a cell using the language of statecharts [16], as implemented in the Rhapsody tool [44]. Separately, we specified a module for structural development using a concept inspired by sweeps to branched skeleton algorithms [33]. Accordingly, an autonomous object serves as the morphogenesis module, which holds predefined skeletons of the structure at various developmental stages. The two modules direct at run time the development of instances of a cell in the simulation. Events that are generated by the molecular module drive the molecular development of cells, which are in parallel directed by the morphogenesis module toward the predefined skeleton.

\footnotetext{
${ }^{1}$ Here, we abuse the term 'divide and conquer', which is generally used in computer science to describe an algorithm design paradigm that works by recursively breaking down a problem into two or more sub-problems of the same (or related) type, until these become simple enough to be solved directly.
} 
We linked the model to a 3D animated front-end (in 3DGameStudio [1]) and an analysis GUI (in MATLAB [23]), using the idea of Reactive Animation [10] and its generic platform [18]. At run-time, the simulation is visualized and analyzed at a front-end and a GUI, respectively.

To analyze the model, we separately examined the behavior of each module. The emerging structure was compared against images of 2D histological sections, while the molecular interactions were compared to a simple mathematical model that describes the kinetics of cell populations. The analysis revealed qualitatively similar results, but we found the overall approach insufficient for realistic modeling of the complete system. In particular, when we attempted to extend the coverage, we had to introduce cross-scaling to capture the interplay between structural development and molecular interactions (i.e., the two independent modules).

\section{Pancreatic Organogenesis}

In mice, pancreatic organogenesis is initiated on the eighth embryonic day, and is roughly divided into two transitions, primary and secondary [29]. During the primary transition, cells in the appropriate regions of the flat gut are specified as pancreatic and form a bud; during the secondary transition, the bud evolves and becomes a branched structure [20, 41]. Organogenesis depends on simultaneous interactions across molecular and morphogenetic mechanisms that act in concert to form the organ. The molecular mechanisms involve processes that regulate the differentiation and development of individual cells (see, e.g., Fig. 1, left), whereas the morphogenic mechanisms gather the cells together to form a cauliflower-like shaped organ (see, e.g., Fig. 1, right). Pancreatic morphogenesis is initiated by a budding process, which leads to thickening of the flat gut. Later, followed by mesenchyme intervention, the pancreas undergoes a process of branching to create the branched structure of the matured pancreas [20]. In parallel, different molecular processes (e.g., proliferation and pancreatic specification) promote the molecular development of the organ $[4,5,20]$. These processes are driven by interactions between different elements including intra-cellular signaling and cell-cell interaction [9, 21, 26, 37].

\section{Methods}

\subsection{Modeling Approach}

We studied the morphogenetic behavior and the molecular interactions in pancreatic organogenesis. Using the language of statecharts [16, 17], as it is implemented in the Rhapsody tool [44], we formalized the molecular interactions of a pancreatic cell. Statecharts define behavior using a hierarchy of states with transitions, events, and conditions. The language can be compiled into executable code using the Rhapsody tool. To promote aggregation of cells toward a specific structure, we employed a concept that directs objects toward a pre-defined fixed skeleton. This concept was 

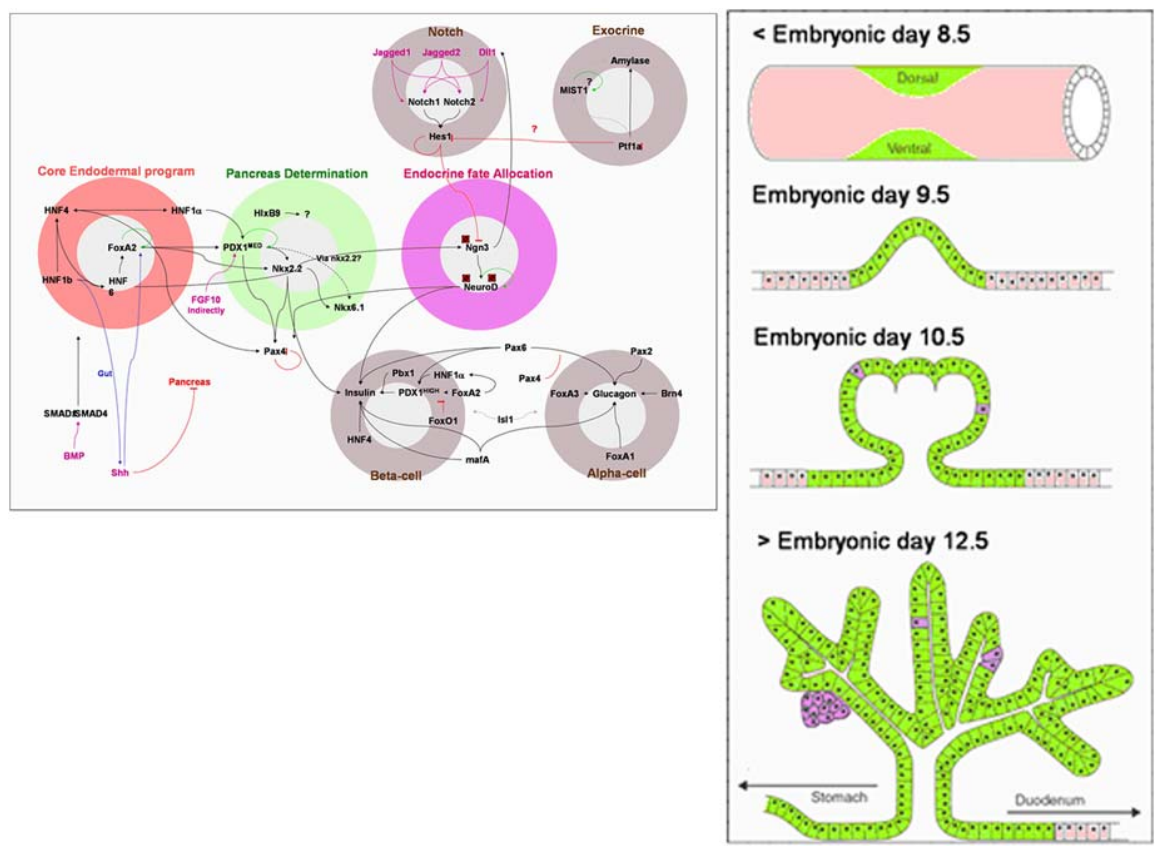

Fig. 1 Left: An illustration of the many molecular interactions driving the pancreatic differentiation in a single cell (adapted from [20]; Reprinted with permission of Wiley-Liss, Inc., a subsidiary of John Wiley \& Sons, Inc.). Right: An illustration of pancreatic formation at four different time points, as depicted in the literature (adopted from [21])

inspired by sweeps to branched skeleton algorithms [25, 33] and was adjusted to pancreatic organogenesis by adding relevant features such as 3-dimensionality and proliferation.

To visualize the model, we used the idea of Reactive Animation [10], a technique that links a reactive executable model with an animated front-end to form a visualized, interactive, and dynamic model. Using a generic platform for Reactive Animation [18], we linked the model to a 3D animated front-end (in 3DGS [1]) and a mathematical GUI (in MATLAB). The front-end visualizes the simulation and provides the means to interact with it, while the mathematical GUI monitors and analyzes the progress. At run time, processes in the simulation are reflected in the front-end in different ways, e.g., by color and position changes. Separately, the mathematical GUI displays various graphs and statistics of the simulation. A prerecorded clip of the simulation at run-time is available at research.microsoft.com/ yakis/runs.

\subsection{Modeling Molecular Interactions}

A eukaryote cell consists of many concurrent sub-cellular and molecular mechanisms that drive development and function over its life cycle. Each sub-cellular 


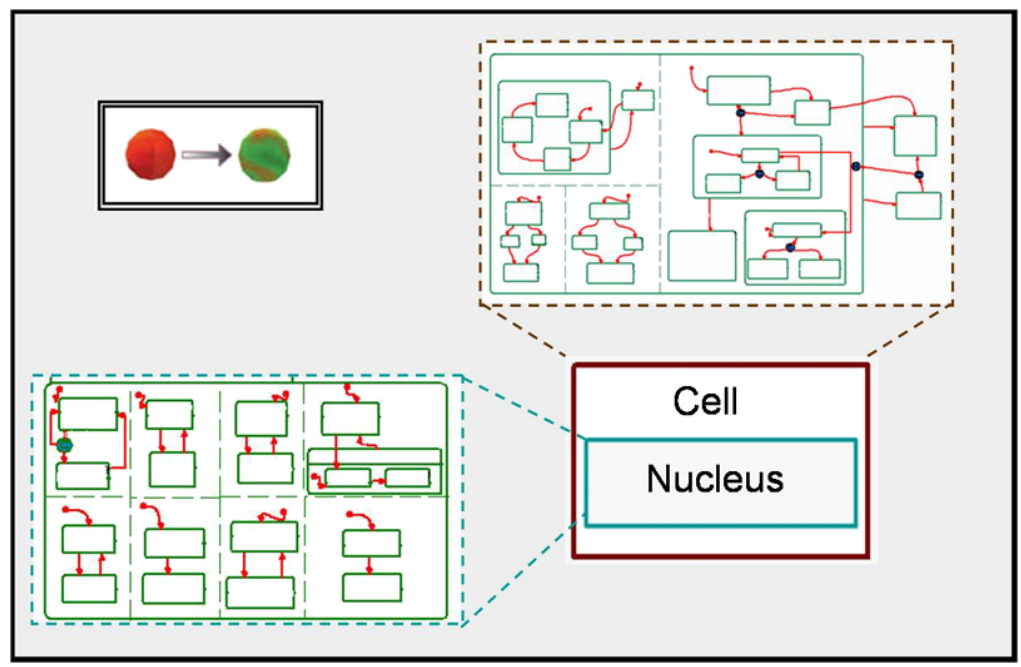

Fig. 2 Modeling eukaryotic cell. The two objects, Cell and Nucleus, accompanied by a pseudo statechart for their behavior

element consists of many concurrent processes and mechanisms that dynamically drive the cell's function over time. In the model, we formalized a cell as an object and its nucleus as a sub-object to indicate (strong) composition between them. Accordingly, the Nucleus object specifies behavior for the gene expression in the cell, while the Cell object itself specifies the behavior of molecular mechanisms (e.g., proliferation). This setup is illustrated in Fig. 2, which shows the cell object accompanied by schematic versions of their statecharts.

The nucleus, the core of a cell, contains the DNA and consists of genes that regulate its development. The genes are expressed in response to various signals in the cell. Genes express proteins that influence the cell's behavior. To model the nucleus, we took a simplistic approach, defining each gene as an independent component that can be either Expressed or Unexpressed. The effect of gene expression is diverse and depends on the process. Gene expression is visualized in different manners. For example, in the case of markers, proteins that determine differentiation, expression is visualized by color changes of the animated cell. In pancreatic organogenesis, one important gene is PDX1, which is considered as the pancreatic marker. In the model, when the active state of the PDX1 component moves to EXpressed the corresponding animated figure changes its color from red to green. The color change indicates that this specific cell accomplished pancreatic specification and is now specified as pancreatic progenitor and no longer as an endodermal cell.

The Cell itself describes the behavior of various molecular mechanisms (such as, differentiation, proliferation, death) in a cell during its lifespan. This element also carries the spatial 3D coordinates of the cell and updates their values at run-time as the simulation progresses. We specify the mechanisms as orthogonal components, 
which at run-time act concurrently to drive the cell's behavior over time. As an example, consider the cell proliferation process. The Proliferation component defines a state for each stage of the cell cycle. At run-time, when the Proliferation ends (i.e., its active state moves to state M), the Cell duplicates itself by creating an identical Cell instance (i.e., a new instance of a cell is created and its active states are set to be identical to its parent). At the front-end, an additional animated cell is created in the appropriate location, which was calculated during its parent division.

\subsection{Modeling Structural Formation}

To model the morphogenetic behavior of cells, we use a concept inspired by sweeps to branched skeleton algorithms (STBS). STBS algorithms are used to model lobed leaves by predefining a branch skeleton to reconstruct the plant structure $[31,33]$. In the past, these algorithms were mainly used to extract the structure of a 2D scanned object. In organogenesis of natural systems, we have to consider a population of objects that, among other things, proliferate and interact. Thus, we adjusted the concept to support morphogenesis of proliferating population in 3D.

As mentioned, among other things, we assigned a property in the Cell object that specifies its spatial coordinates. Accordingly, when a newborn $\mathrm{Cell}$ is initiated, its spatial properties are updated based on its parent location. In addition, we defined another object, which utilizes pre-defined 3D skeletons that determines generic structures of the organ at different stages. This STBS object corresponds to mechanisms in the environment that promote cells to aggregate and form the pancreatic structure. At run time, the object directs cells by minimizing the distance between the cell the skeleton. Thus, cells are 'swept' toward the skeleton to form the branched structure of the pancreas. At the front-end, the animated cells continuously update their positions. As the animation progresses, the visualization discloses how the population aggregates to form the branched structure of the pancreas.

\subsection{Combining the Two: The Simulation at Run-Time}

When the model is executed, instances of the Cell (Fig. 2) are created and appear in the front-end as a sheet of red spheres on the proper location at the flat endodermal Gut. Once a Cell instance is created, one state in each concurrent component of the statechart is set to be an active state. At this point, the Cells are uniform and their active states are set to the initial states (designated by a stubbed arrow). As the simulation advances, cells respond to various signals by changing their active states accordingly. Hence, the sheet loses uniformity at a very early stage of the simulation.

To illustrate the simulation in progress, consider a conceptual process that unifies many biological processes such as signaling pathways or cell migration. Such 
a process is stimulated by a signal that initiates a chain of various reactions. Consequently, events are sent to the Nucleus, which initiates expression in various Genes. In turn, the active state in the relevant components moves to the Expressed state and the corresponding animated cell changes its color. Eventually, an event is generated and the relevant molecular mechanisms move to new states. For example, an event may promote a cell to proliferate, and thus the active state in the Proliferation component in the Cell becomes M. Consequently, the cell duplicates itself, and a new animated cell appears in the front-end. In parallel, cells are specified as pancreatic and change the color of their corresponding spheres (Fig. 2 top-left).

At the same time, the STBS object directs cell movement towards the predefined skeleton. Accordingly, the spatial properties of cells are continuously updated to simulate cell migration. At the front-end, animated cells change their location accordingly to form the pancreatic branched structure. When a cell proliferates, the new instance interacts with the STBS object and is directed based on its parent position. Other molecular mechanisms, such as differentiation or specification, act concurrently. The simulation achieves equilibrium when cells are differentiated and are located in a proper position on the skeleton, where they cannot proliferate anymore.

\section{Results}

\subsection{Behavior of Population Fits Simple Mathematical Model}

To analyze the molecular development of the cell population in the simulation, we recorded the cell count of the endodermal and pancreatic populations over time. Each run of the simulation generated a slightly different growth, but the overall course maintained similar characteristics. Figure 3 (left) shows a typical time dependent molecular development of endodermal (red), pancreatic (green), and overall cell population (black).

We observed three major regimes. In the primary regime (embryonic day 8-9) endodermal cells proliferate but do not specify as pancreatic. Thus, the endodermal population increases but the pancreatic one does not. At the secondary regime, specification signals are introduced and endodermal cells start specifying as pancreatic cells and the proliferation continues in both. In this regime, we observed decrease of the endodermal population and a rapid growth in the pancreatic one. In the last regime (starts approximately at embryonic day 13), both populations enter a steady state, in which the endodermal population is fully specified and the pancreatic population achieved maximum.

To test the plausibility of the results, we constructed a simple mathematical model that simulates the molecular behavior of cell populations in the model. We formalized the system using ordinary differential equations (see, e.g., [8, 38, 39]) to describe the kinetics of cell population. The model (1) considers a population of endodermal cells $(E)$ and a population of pancreatic cells $(P)$. E proliferates at rate 

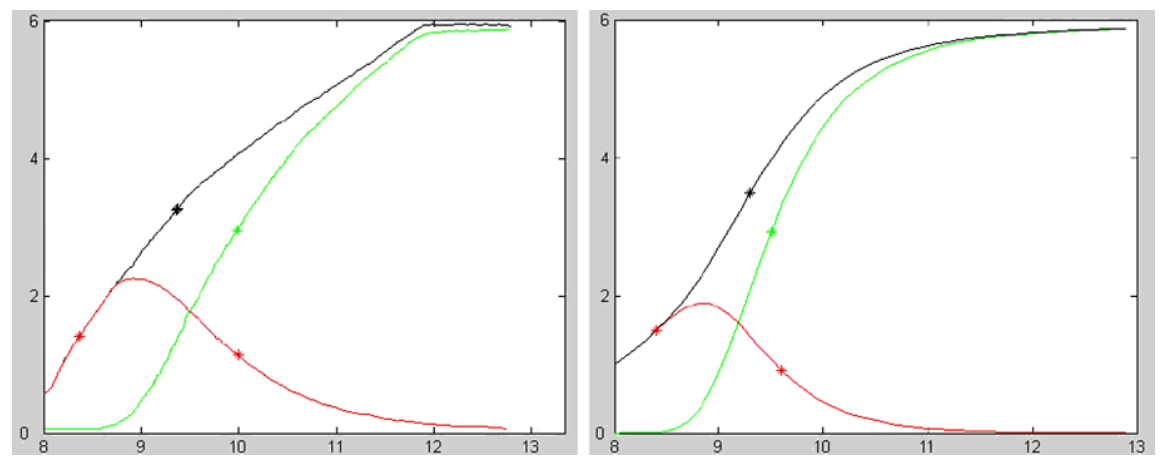

Fig. 3 Molecular development in pancreatic organogenesis: Cell count (in thousands) of endodermal (red), pancreatic (green) and overall (black) population as function of time (in embryonic days). (Notice the star symbol, indicating the median values.). Left: Cell count in the computational statechart-based model. Right: Mathematical ODE-based model with the parameters $k_{p}=1$, $k_{s}=3, f_{p}=4 /\left(t^{n}+4\right)$ and $f_{s}=t^{n} /\left(t^{n}+1\right)$

$k_{p}$ and specifies as pancreatic in rate $k_{s}$, which is regulated by additional signals formalized in $f_{s}$. The pancreatic population $P$ is increased by the specification and proliferation processes. Pancreatic cells proliferate at the same rate an endodermal but are bounded by additional signals, thus we assign the $f_{p}$ element to describe effect of such. Figure 3 (right) shows a solution for the mathematical model. This result manages to reproduce the behavior of the cell population and the three regimes described above.

$$
\begin{aligned}
& d E / d t=k_{p} E-k_{s} f_{s} E, \\
& d P / d t=k_{p} f_{p} P+k_{s} f_{s} E .
\end{aligned}
$$

The difference between the two models emerges from the modeling perspective. While the mathematical model describes the behavior of cells as a population, the computational model specifies behavior of an individual cell and the behavior of the population emerges from execution of many instances with the same specification. Although simplified, the mathematical model revealed similar characteristics for the populations as emerged from the computational simulation. Here, we used the mathematical model for qualitative analysis only and did not examine the model in detail. However, a quantitative analysis is possible, but is beyond the scope of this paper.

\subsection{The Emerging Structure Generates Histological Sections}

As the simulation advances, cells proliferate and are directed by the morphogenesis module toward the branched structure of the pancreas. Figure 4 shows four steps in the formation of the structure from flat sheet into the matured pancreas 


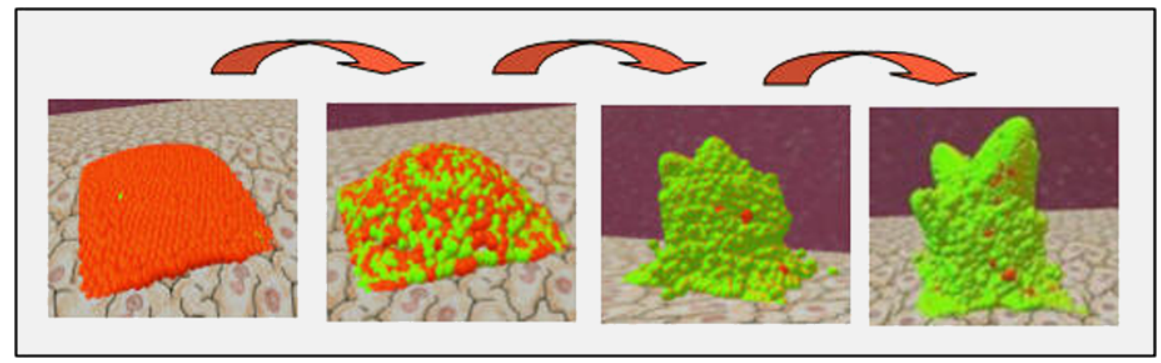

Fig. 4 Four snapshots of the structure formation in the simulation: endodermal (red) and pancreatic (green) cells act in concert to form the branched pancreatic structure

Fig. 5 Comparison of the emerging structure and 2D histological sections at embryonic day 10 (top; adapted from [20]) and 10.5 (bottom; contributed by Dor's lab)

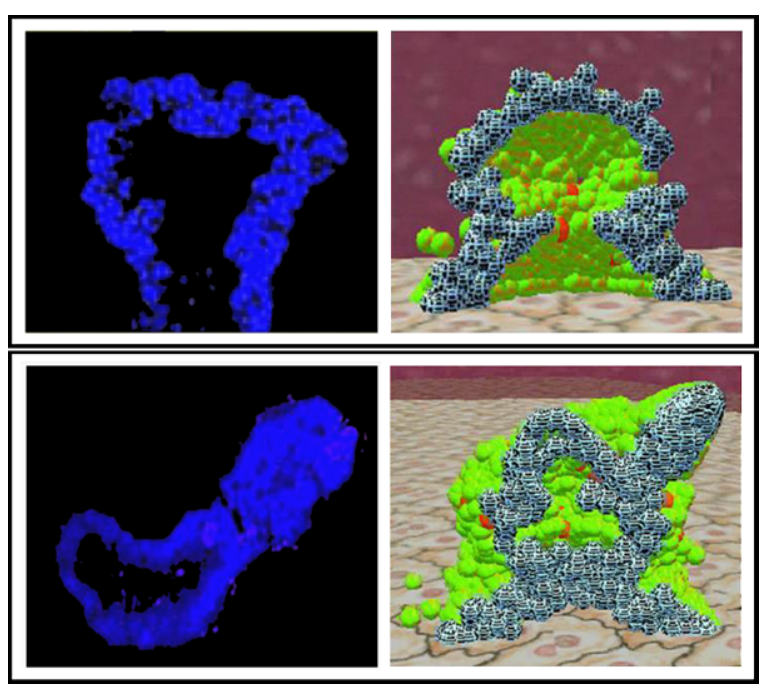

(see a recorded clip at research.microsoft.com/ yakis/branching). To compare the 3D emerging structure with the two dimensional data (e.g., Fig. 1, right), we enabled a 'halt and slice' option in the animated front-end. Accordingly, at any stage of the simulation, the user can halt the simulation and view slices of the emerging structure over the $x$-and the $y$-axis. The cross-section slices are then compared with the histological section. A comparison of 2D histological sections (left) and cross sections of the simulated structure (right) is shown in Fig. 5. The results indicated that the emerging structure manages to qualitatively illustrate somewhat of the dynamic of early stages of pancreatic morphogenesis. Notice that in addition to the structural formation, the molecular behavior is also visualized in the front-end at run time. For example, cells proliferate by creating new instances and change their properties (e.g., color) to indicate gene expression. It is important to emphasize that the molecular changes are not effected by the cell position, which is being regulated separately. 


\subsection{The Need for Cross-scaling in Pancreatic Organogenesis}

As mentioned, the 'divide and conquer' approach, by its very nature, separates the molecular interactions from the structural formation. Thus, it does not conduct the interplay between main modules. To proceed with realistic modeling of pancreatic organogenesis, in particular to advance to the 'secondary transition' in which cells adopt their final fate, we had to introduce cross-scaling [7] to enable interplay between the molecular interactions and the structural formation.

The principles underlying cross-scaling in modeling natural systems are illustrated in Fig. 6. In the 'divide and conquer' approach (Fig. 6, top), a module for molecular interactions (left) is separated from the structural behavior (right). At run time, the two modules interact with cells (middle), to drive in parallel their molecular and structural development. An approach that considers cross-scaling (Fig. 6, bottom) links the two modules and enables interplay between them. At run time, molecular interactions and structural information direct cell development as before, but also interact with each other to promote different aspects in their development.

The Delta-Notch mechanism emphasizes the need for cross-scaling in organogenesis. In pancreatic organogenesis, this mechanism directs cells towards their final fate. It is partly initiated by external signals (from the extra-cellular space) and is enhanced by cell-cell interaction. At the same time, cells that adopted an endocrine fate, aggregate to form clusters named 'islet of Langerhans'. In this case, the stage of a cell is significantly determined by external signals in its spatial position. Thus, the molecular development relies much on morphogenesis. In turn, a cell that adopted an endocrine fate, starts aggregating in a cluster, and thus the molecular interactions determine the structural formation. Such a mechanism forces cross-scaling for simulating its behavior.

Fig. 6 Top: An illustration of the interaction scheme between the cells and the morphogenetic and molecular interactions in the model. Bottom: Cross-scaling in modeling (notice the extra arrow indicating interactions between the modules)

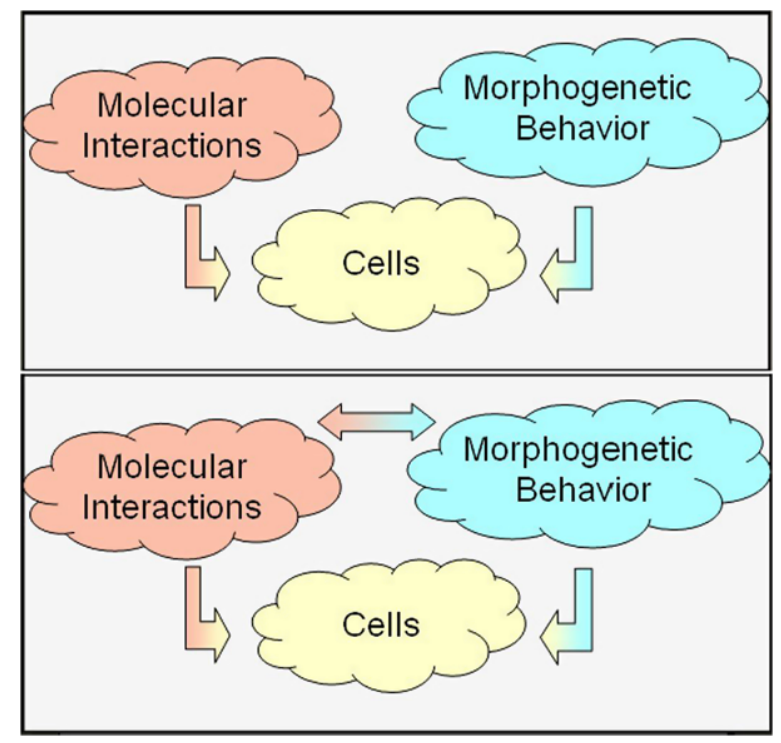




\section{Discussion}

During the last decade, increasing interdisciplinary work modeled aspects in natural systems to explain relevant experimental findings (see, e.g., [2, 6, 14, 27]). However, most of this work describes the system at a specific scale and ignores others. Moreover, the work focuses on a single mechanism in each scale and disregards multiple interactions. Another type of modeling work formalizes gene expression and protein activity using a variety of mathematical and computational tools (for example, see $[3,19,30,34,35])$. However, most of the relevant work ignores multiple concurrent activities and focuses on a single mechanism in the entire system. An example of comprehensive modeling is the beating heart project [28], which over the last fifty years formalized the electric activities in the heart. However, by its mathematical nature, the model is not interactive and does not support the kind of visualization we seek.

Recently, various papers use computational modeling approaches for natural systems. In [13], hybrid automata are used to model the Delta-Notch mechanism, which directs differentiation in various natural systems. In [11], computational challenges of systems biology are described and various approaches for achieving them are discussed. A similar motivation for model-driven engineering approaches is discussed in [36]. In [12], computational and mathematical approaches are reviewed and the term executable biology is used to describe the kinds of modeling carried out in our group, and recently also elsewhere. In [45], a model for a eukaryotic cell is built, in which a UML class diagram was used to formalize the relations between a cell and its sub-cellular elements. The setup was empowered by specifying behavior of different cell types (e.g., red blood cell) using the ROOM formalism. A similar approach was employed in [43] to model the Ethylene-Pathway in Arabidopsis thaliana using statecharts and LSCs.

To simulate the development of natural systems, we confront a complex system that dynamically evolves over time and space. This task requires one to understand the many time-dependant aspects of the system. A comprehensive modeling approach of such systems should provide the means to specify behavior of its different aspects [7]. Moreover, the approach should deal with the system on different levels of abstraction, which in turn drive different scales of the development. At the very least, such an approach should provide the means to simulate the dynamics of formation of a 3D structure.

The 'divide and conquer' approach presented here was inspired by many industrial reactive systems, which can be separated into fundamental components that interact at run time. In natural systems, this approach provides the means to specify dynamic behavior of the structural and molecular development of a natural system as separated modules. From the biological point of view, these modules correspond to different scales of the system, organ, cell, gene, etc. However, by its nature, the approach does not provide the means to specify interplay between the different modules. Thus, it conducts multi-scaling and 3D dynamics but avoids cross-scaling.

In this paper, we describe how the 'divide and conquer' approach was employed for modeling pancreatic organogenesis. The end result gave rise to dynamic rep- 
resentation of the system and managed to capture the preliminary stages of its development. Furthermore, the model provided plausible results for the preliminary period. However, more complex mechanisms of the system could not be specified using this approach. These mechanisms involve interplay between structural and molecular behaviors, which are specified separately in the model. Thus, we move that this approach can be used to model such systems as the development of phyllotaxis in plants, which might be separated into fundamental modules. For example, structural phyllotaxis principles can be specified as L-Systems [22, 32], while molecular behavior can be formalized using deferential equations. Furthermore, existing models (such as, e.g., $[15,24,42]$ ), which describe structural formation of natural systems, can be extended using the 'divide and conquer' approach to support molecular behavior.

In the case of pancreatic organogenesis, the 'divide and conquer' approach emphasized the need for cross-scaling in organogenesis, and served as a significant milestone in our work toward the model described in [40], which employs the 'autonomous cell' approach.

Acknowledgements We thank Yuval Dor and Judith Magenheim for providing histological images of the pancreas.

\section{References}

1. 3D Game Studio. www.3dgamestudio.com

2. Axelrod JD (2006) Cell shape in proliferating epithelia: a multifaceted problem. Cell 126:643645

3. Cardelli L (2005) Abstract machines of systems biology. Trans Comput Syst Biol 3:145-168

4. Chakrabarti SK, Mirmira RG (2003) Transcription factors direct the development and function of pancreatic beta cells. Trends Endocrinol Metab 14:78-84

5. Chu K, Nemoz-Gaillard E, Tsai MJ (2001) BETA2 and pancreatic islet development. Recent Prog Horm Res 56:23-46

6. Ciliberto A, Novak B, Tyson JJ (2003) Mathematical model of the morphogenesis checkpoint in budding yeast. J Cell Biol 163:1243-1254

7. Cohen IR, Harel D (2007) Explaining a complex living system: dynamics, multi-scaling and emergence. J R Soc Interface 4:175-182

8. Edelstein-Keshet L (2005) Mathematical models in biology. Society for Industrial and Applied Mathematics, Philadelphia

9. Edlund H (2002) Pancreatic organogenesis-developmental mechanisms and implications for therapy. Nat Rev Genet 3:524-532

10. Efroni S, Harel D, Cohen IR (2005) Reactive animation: realistic modeling of complex dynamic systems. IEEE Comput 38:38-47

11. Finkelstein A, Hetherington J, Li L, Margoninski O, Saffrey P, Seymour R, Warner A (2004) Computational challenges of systems biology. IEEE Comput 37(5):26-33

12. Fisher J, Henzinger TA (2007) Executable cell biology. Nat Biotechnol 25:1239-1249

13. Ghosh R, Tomlin C (2004) Symbolic reachable set computation of piecewise affine hybrid automata and its application to biological modelling: delta-notch protein signalling. Syst Biol (Stevenage) 1:170-183

14. Gibson MC, Patel AB, Nagpal R, Perrimon N (2006) The emergence of geometric order in proliferating metazoan epithelia. Nature 442:1038-1041 
15. Gorgevik D, Loskovska S, Mihajlov D (1994) Lindenmayer system application on the human kidney arterial system. In: Proceedings of the 12th international congress of the European federation for medical informatics, pp 127-131

16. Harel D (1987) Statecharts: a visual formalism for complex systems. Sci Comput Program 8:231-274

17. Harel D, Gery E (1997) Executable object modeling with statecharts. IEEE Comput 30:31-42

18. Harel D, Setty Y (2007) Generic reactive animation: realistic modeling of natural complex systems (submitted)

19. Heath J, Kwiatkowska M, Norman G, Parker D, Tymchyshyn O (2006) Probabilistic model checking of complex biological pathways. In: Priami C (ed) Proceedings of the computational methods in systems biology (CMSB'06). Lecture notes in bioinformatics, vol 4210. Springer, Berlin, pp 32-47

20. Jensen J (2004) Gene regulatory factors in pancreatic development. Dev Dyn 229:176-200

21. Kim SK, MacDonald RJ (2002) Signaling and transcriptional control of pancreatic organogenesis. Curr Opin Genet Dev 12:540-547

22. Lindenmayer A (1968) Mathematical models for cellular interaction in development. J Theor Biol 18:280-315

23. The MathWorks. www.mathworks.com

24. Mundermann L, Erasmus Y, Lane B, Coen E, Prusinkiewicz P (2005) Quantitative modeling of arabidopsis development. Plant Physiol 139:960-968

25. Mundermann L, MacMurchy P, Pivovarov J, Prusinkiewicz P (2003) Modeling lobed leaves. In: Proceedings of computer graphics international

26. Murtaugh LC, Melton DA (2003) Genes, signals, and lineages in pancreas development. Annu Rev Cell Dev Biol 19:71-89

27. Nelson CM, Vanduijn MM, Inman JL, Fletcher DA, Bissell MJ (2006) Tissue geometry determines sites of mammary branching morphogenesis in organotypic cultures. Science 314:298 300

28. Noble D (2005) The heart is already working. Biochem Soc Trans 33:539-542

29. Pictet RL, Clark WR, Williams RH, Rutter WJ (1972) An ultrastructural analysis of the developing embryonic pancreas. Dev Biol 29:436-467

30. Priami C, Quaglia P (2004) Modelling the dynamics of biosystems. Brief Bioinform 5:259269

31. Prusinkiewicz P (2004) Modeling plant growth and development. Curr Opin Plant Biol 7:7983

32. Prusinkiewicz P, Hanan J (1989) Lindenmayer systems, fractals and plants. Springer, New York

33. Prusinkiewicz P, Rolland-Lagan AG (2006) Modeling plant morphogenesis. Curr Opin Plant Biol 9:83-88

34. Regev A, Shapiro E (2002) Cellular abstractions: cells as computation. Nature 419:343

35. Regev A, Silverman W, Shapiro E (2001) Representation and simulation of biochemical processes using the pi-calculus process algebra. In: Pacific symposium on biocomputing, pp 459-470

36. Roux-Rouquié M, daRosa DS (2006) Ten top reasons for systems biology to get into modeldriven engineering. In: GaMMa'06: proceedings of the 2006 international workshop on global integrated model management. New York, ACM, pp 55-58

37. Schonhoff SE, Giel-Moloney M, Leiter AB (2004) Minireview: development and differentiation of gut endocrine cells. Endocrinology 145:2639-2644

38. Segel LA (1984) Modeling dynamic phenomena in molecular and cellular biology. Cambridge University Press, New York

39. Segel LA (1991) Biological kinetics. Cambridge University Press, New York

40. Setty Y, Cohen IR, Dor Y, Harel D (2008) Four-dimensional realistic modeling of pancreatic organogenesis. Proc Natl Acad Sci USA 105(51):20374-20379

41. Slack JM (1995) Developmental biology of the pancreas. Development 121:1569-1580

42. Smith RS, Guyomarc'h S, Mandel T, Reinhardt D, Kuhlemeier C, Prusinkiewicz P (2006) A plausible model of phyllotaxis. Proc Natl Acad Sci USA 103:1301-1306 
43. Taubner C, Merker T (2005) Discrete modelling of the ethylene-pathway. In: ICDEW'05: proceedings of the 21 st international conference on data engineering workshops. Washington, IEEE Computer Society, p 1152

44. Telelogic. www.telelogic.com

45. Webb K, White T (2006) Cell modeling with reusable agent-based formalisms. Appl Intell 24(2):169-181 